\title{
Student Nurses' Experiences of The Clinical Psychiatric Learning Environment in an Education Institution
}

\author{
Miriam Moagi \\ Elsie Janse van Rensburg \\ University of Pretoria \\ Jeanette Maritz \\ University of South Africa
}

Address correspondence to Dr. Elsie Janse van Rensburg, Department of Health Studies, University of South Africa, PO Box 392 , Pretoria, 0003. E-mail: jvrenes@unisa.ac.za

This study investigated lived experiences of student nurses working in a clinical psychiatric learning environment in South Africa. Participants were 29 fourth-year student nurses in the clinical psychiatric learning environment at a nursing education institution (females = 27; males =2). They engaged in focus group discussion on their experiences of the clinical psychiatric learning environment. The data were thematically analyzed using Tesch's method of open coding. The student nurses experienced the environment as growth enhancing on a professional and personal level.

Keywords: Student nurses, psychiatric nursing, clinical learning environment, mental health, qualitative research

Clinical learning plays an important role in nursing education, as it provides opportunities for student nurses to apply the theory learned in the classroom to the real world of clinical nursing (Hayman-White \& Happel, 2005). It is also a socialisation process through which student nurses are inducted into the practices, expectations and real-life work environment of the nursing profession. Clinical education is a vital component of psychiatric nursing science, as it provides student nurses with the opportunity to combine cognitive, psychomotor and affective skills, thus gaining knowledge, skills and experience in the clinical psychiatric learning environment. Chan (2001) found that clinical education involves clinical practice and is a multidimensional entity with a complex social context that allows student nurses to consolidate their knowledge and practice skills by using it effectively and productively.

Secomb (2008) states that it is important for student nurses to have access to the "real-world situations" (clinical placements) in order to apply their skills, knowledge and attitudes, which enable a level of competence during clinical practice. Student nurses often perceive the psychiatric nursing placement as a stark contrast to the general nursing settings (Charleston \& Happel, 2006). This view on psychiatric nursing placement is supported by Sharif and Masoumi (2005), who added that thirdand fourth-year nursing students reported that the clinical psychiatric learning environment was the most stressful part of the nursing programme, as it involves unfamiliar areas and difficult psychiatric patients. According to Van Rhyn and Gontsana (2004), student nurses are confronted with unique challenges in a clinical psychiatric learning environment. They have to deal, for example, with mental health care users (MHCUs) who can become physically violent or demonstrate unpredictable and/or suicidal behaviour. They also have difficulty in differentiating between their roles of both student and nurse (Chan 2001).

A clinical learning environment that is rich in learning experiences, but lacks a supportive environment, discourages the student nurses to seek experience, which results in the loss of learning and growth opportunities (Mabuda, Potgieter \& Alberts, 2008). In a study focusing on the clinical experience of student nurses, Van Rhyn and Gontsana (2004) found that the theory and practice in psychiatric nursing for student nurses, while training, causes severe stress due to poor integration of theory and practice. Student nurses may experience anxiety, uncertainty and feelings of abandonment when first exposed to the complex health care environment (Chesser-Smyth, 2005).

The nature of the clinical psychiatric learning environment refers to the unpredictable behaviour of MHCUs. Nursing students often associate the unpredictable behaviour of MHCUs with closed wards in the mental health facility. A closed ward is a restricted environment to provide $\mathrm{MHCU}$ s with care, treatment and rehabilitation services with closer supervision and monitoring to minimise the risk of danger to self, others or property. MHCUs are admitted to closed wards when they relapse and have acute symptoms which include psychosis and hallucinations. Support provided during exposure to the clinical learning environment may generate a positive attitude in student nurses towards psychiatric nursing. A positive attitude may facilitate the promotion of these students' mental health by improving their self-confidence and self-esteem (Pillay \& Mtshali, 2008).

\section{Goals of the Study}

The study explored student nurses' work experiences in order to better understand how best to promote their mental health in the work setting. The research question was:

What are the student nurses' work experiences when working in the clinical psychiatric learning environment and why is it important for their mental health?

\section{Method}

\section{Participants and Setting}

Participants were 29 student nurses. Table 1 presents the participant characteristics. 
Table 1

Participant Characteristics

\begin{tabular}{lll}
\hline Race & Africans: 28 & White: 1 \\
Gender & Females: 27 & Males: 2 \\
Ages & $21-40$ years & \\
Total number of participants & 29 & \\
\hline
\end{tabular}

\section{Data Collection}

Participants were asked to complete drawings and naïve sketches before starting with the focus group interviews. This assisted participants to think about their experiences and created awareness for them during the focus group interviews. Field notes were written during and after data collection. A total of three focus group interviews were conducted with the student nurses in the nursing education institution. Each focus group interview lasted 30 to 60 minutes and was audio-recorded and transcribed verbatim with all the participants' permission. The focus group interviews each comprised nine to 11 members. The following two in-depth questions were asked during focus group interviews: "What is it like for you to work in the clinical psychiatric learning environment?" and "What can be done to enhance your mental health in the clinical psychiatric learning environment?"

Triangulation was obtained by using multiple methods of data collection, namely, drawings, naïve sketches, focus group interviews and field notes. Dependability was maintained through code-recode procedures.

\section{Procedure}

Written and informed consent was obtained before the collection of data commenced. Participation was voluntary.

\section{Data Analysis}

Data were thematically analyzed. The process of data analysis, that is reading of transcripts, drawings, naïve sketches, focus group interviews and field notes, was done by the researcher. Participants explained their own viewpoint of the drawings. The steps of data analysis were followed as described by Tesch's method of open coding (cited in Creswell, 2003). Themes, categories and sub-categories were identified and controlled with literature. An independent coder was used to enhance trustworthiness.

\section{Results and Discussion}

Student nurses' profession and personal growth experiences; and student nurses experienced a "life-changing" learning process that filtered through to their real-life context. Each will now be discussed with the related sub-themes. Verbatim quotes are in italics.

\section{Student Nurses' Profession and Personal Growth Experiences}

The student nurses had a deeper appreciation of the complexity of the mental health care environment, and also its realism or ordinariness. The following statements are illustrative:

Before being exposed to the mental health care facility, I always believed that people with mental illness are bewitched because they are very intelligent. I couldn't wait to come to a mental health facility to see those people. (Participant \# 6).

Before I came to the mental health facility, I thought that people with mental illness you cannot live with them. Be- cause in my community most of them are isolated and they are being abused... What I have also learned is that most of the people with mental disabilities did not choose to become like that. (Participant \# 28).

Madianos, Priami, Alevisopoulos, Koukia and Rogakou (2005) were of the view and add that in mental health, clinical placement plays an important role in nursing education by providing student nurses with their first real insight into the nature, role and responsibilities of nursing in the mental health context.

Student nurses, prior to exposure to MHCUs in the clinical learning environment, were unsure of how they would cope with caring for people with mental illness. The uncertainty and anxiety was reflected in the field notes.

\section{A participant said:}

It was like at first I asked myself how am I going to cope you know, cause I thought nursing is like giving people treatment you know and taking of temperature and all sort of things, so I never thought that you can talk to somebody as part of treatment, so it was very complex for me, I have to learn those procedures like interviewing people, sometimes they talk and change the subject, so it was very complex like in the beginning, but you can see every time you are going there it was very challenging for me. (Participant \# 4).

The initial anxiety experienced by these student nurses led to different perceptions of MHCUs.

For me, in the community when you see this mentally unstable person, and when you come to a mental health care facility, they are mad, so you are thinking that everybody go around stripping naked and when we meet them they are going to beat us. You think of all those things that this people are aggressive they are violent, so you are thinking of my goodness how am I going to deal with them.(Participant \# 13).

I was scared of them at first because of the stories I heard from the community...they can do this and that to you, or they can harm you... (Participant \# 9).

In her study on student nurses working with mentally challenged individuals, Janse van Rensburg (2010) confirmed the uncertainty about the student nurses' ability to cope. The students in her study experienced the first exposure as a "new experience" that created emotional discomfort for them.

The nature of the clinical psychiatric learning environment. For the purpose of this article, MHCUs with acute symptoms often display physical and verbal aggression, which student nurses witness during their placement in this clinical psychiatric learning environment.

Where I was placed there was this mental health care user... was so angry that she would not allow anyone to enter the ward, she blocked the door, she was suppose to be taken off the door, she was so dangerous, she could use anything that she get in her hand to assault you, I was scared and traumatised by that behaviour. (Participant \# 15). 
When MHCUs relapse, they can become aggressive (verbally or physically) towards the student nurses.

When they relapse, this mental health care users was very aggressive, they were eating and he wanted to hit us, he was throwing chairs and all that at us, I was afraid that what if that chair can hit one of us, what is going to happen". (Participant \# 5).

The role of nursing staff in the learning context. In this study student nurses came to the clinical psychiatric learning environment with theoretical knowledge, but lacking practical experience. Nursing staff in the clinical psychiatric learning environment play an important role in the learning context of the student nurses, by guiding, supporting and socialising them into the field of psychiatric nursing.

Student nurses experienced a negative attitude from nursing staff as they were critical and lacked a positive attitude towards the student nurses

The attitude of the nursing staff need to be positive towards the student nurses, because you find that when we come in the ward as students, the staff will complain, and compare us with the previous students who came before us; they do not motivate us to correct the previous mistakes done by student nurses. (Participant \# 19).

Nursing staff in the wards hardly sit with us, no one gave us information about the type of mental health care users in their ward, we spend most of the time alone not knowing what to do, playing games with mental health care users that we do not know, and it was scary. (Participant \# 2).

In a study conducted by O'Brien, Buxton, and Gilles (2008, p.505) on improving the undergraduate clinical placement experience in mental health nursing, clinical nurses are central to students' learning, and students "value the teaching input of nursing staff, therefore consider activities such as explaining and questioning to be integral to their learning". This teaching input provided by nursing staff to student nurses might improve their experience, understanding and knowledge of psychiatric nursing. Fisher (2002) adds that providing a supportive environment, in which learning can take place, means providing students with the needed assistance to process their experiences. Clinical placements provide opportunities for professiona socialisation by allowing student nurses to experience how al categories of staff members interact, feel and think about what they value and how they communicate (Levett-Jones \& Lathlean, 2008).

\section{Student Nurses Experienced a "Life-Changing" Learning Process That Filtered Through to the Real-Life Context of the Student}

The student nurses described their "life-changing" process as resulting in growth on multiple levels (spiritual, psychological, social and physical). Students experienced growth and adaptation and moved from an initial sense of feeling psychologically unsafe and anxious to a position where they experienced a greater sense of safety and positive emotive responses. The second theme will be discussed by referring to an attitudinal shift (looking through new lenses) and actualising learning in the real-life context.

Attitudinal shifts: Looking through new lenses. By the completion of their course, student nurses had overcome their initial anxiety of working with MHCUs and many expressed a positive change in attitude towards psychiatric nursing. The positive change reflected in the field notes as the facial expressions of participants became more relaxed.
I have learned to appreciate and see life from a different angle, where I come from we used to call them that they are mad. (Participant \# 13).

Student nurses started looking through new lenses when they applied their knowledge and skills in the clinical learning environment. They realised that mental illness can be treated.

When I started working with mental health care users. I started to realise that not all mental health care users are dangerous, unless if they have relapsed, their condition can be treated and managed. (Participant \# 28).

The student nurses' positive attitudes towards psychiatric nursing, as well as MHCUs in the clinical psychiatric learning environment, improved due to the positive clinical experiences and exposure to the relevant theory and practice (Hayman-White \& Happel, 2005). Hayman-White and Happel (2005) further state that positive placement experiences have a positive influence on student nurses' confidence. This positive attitude towards psychiatric nursing and MHCUs might improve students' learning in the real-life context.

Actualising learning in the real-life context. Student nurses experienced a "life-changing" learning process, which resulted in them actualising learning in the real-life context. Student nurses felt they benefited from their exposure to the clinical learning environment as they acquired life skills. These skills could be applied to the communities where they lived.

You are able to live within the community even with people with different personality, you are able to handle them, and it is really beneficial. (Participant \# 10).

Student nurses were able to apply the skills they obtained in psychiatric nursing to their real-life context.

A participant said:

I have learned that it is our duty as psychiatric nurses to go back to our community to try to make awareness campaigns, or health talks about mental illness because most of us we not aware of small things that can stress us. So I want us to go out there and help our community. (Participant \# 6).

A study by Janse van Rensburg (2010) confirmed that student nurses who worked with mentally challenged individuals went through a process of personal transformation. This process of personal transformation touched their lives by enriching three dimensions, namely the emotional, spiritual and interpersonal dimensions. In this study, student nurses acquired lifelong learning skills by showing growth and adaptation in that they have gained knowledge and skills and moved from an initial sense of anxiety towards feeling empowered. The psychiatric nursing practice will be improved as a result of the facilitation of the promotion of the mental health of the student nurses in the clinical psychiatric learning environment.

\section{Implications for Practice}

Psychiatric nurse educators should provide guidance and support to student nurses in the clinical psychiatric learning environment by allowing them to have access to information about procedures related to psychiatric nursing practices, and to practise according to standards set by statutory bodies such as nursing councils. Educators should mobilise resources to promote an atmosphere of training and learning. They should encourage student nurses to participate in in-service training, peer mentoring and academic programmes related to working with MHCUs in the clinical psychiatric learning environment. Evidence-based practice and research should be encouraged. Student nurses should have access to research reports related to the experiences of student nurses when working in the clini- 
cal psychiatric learning environment during training so that they may prepare themselves emotionally.

\section{Conclusion}

Student nurses experienced the clinical learning environment as a growth enhancer on a professional and personal level. The exposure to the clinical learning environment developed the student nurses' life skills that could be applied to other areas of their life. The clinical psychiatric learning environment plays an important role in psychiatric nursing education, as it provides opportunities for student nurses to apply the theory learned in the classroom to the real world of clinical psychiatric nursing. Enhancing the guidance and support provided by nurse educators to the student nurses during exposure to the clinical psychiatric learning environment could assist in generating a positive attitude towards psychiatric nursing science. This positive attitude could facilitate the promotion of these student nurses' the mental health by improving their self-confidence and self-esteem. A Limitation in this study was that only two male student nurses volunteered to participate. Future studies might explore how gender can affect the experiences of student nurses in the clinical learning environment.

\section{References}

Chan, D. (2001). Combining qualitative and quantitative methods in assessing hospital learning environments. International Journal of Nursing Studies, 38, 447-459.

Charleston, R., \& Happel, B. (2006). Recognising and reconciling differences: Mental health nurses and nursing students' perceptions of the preceptorship relationship. Australian Journal of Advanced Nursing, 24(2), 38-43.

Chesser-Smyth, P. A. (2005). The lived experiences of general student nurses on their first clinical placement: A phenomenological study. Nurse Education in Practice, 5, 353-359.

Creswell, J. W. (2003). Research design: Qualitative \& quantitative approaches. Thousand Oaks, CA: Sage.

Fisher, J. E. (2002). Fear and learning in mental health settings. International Journal of Mental Health Nursing, 11, 128-134

Hayman-White, K., \& Happel, B. (2005). Nursing students' attitudes towards mental health nursing and consumers: Psychometric properties of a self-report scale. Archives of Psychiatric Nursing, 19(4),184-193.

Janse van Rensburg, E. S. (2010). A model to facilitate the mental health of student nurses working with mentally challenged individuals (Unpublished doctoral thesis). University of Johannesburg, Johannesburg, South Africa.

Levett-Jones, T., \& Lathlean J. (2007). Belongingness: A prerequisite for nursing students' clinical. Nurse Education in Practice, 36, 103-111.

Mabuda, B. T., Potgieter, E., \& Alberts, U. U. (2008). Student nurses experiences during clinical practice in the Limpopo Province. Curationis, 3(1), 19-27.

Madianos, M. G., Priami, M., Alevisopoulos, G., Koukia, R., \& Rogakou, E. (2005). Nursing students' attitude change towards mental illness and psychiatric case recognition after a clerkship in psychiatry. Mental Health Nursing, 26, 169-183.

O'Brien, L., Buxton, M., \& Gilles, D. (2008). Improving the undergraduate clinical placement experience in mental health nursing. Issues in Mental health Nursing, 29, 505-522.
Pillay, P., \& Mtshali, N. G. (2008). Clinical supervision and support for bridging programme students in the greater Durban area. Curationis, 31(4), 46-56.

Secomb, J. (2008). A systematic review of peer teaching and learning in clinical education. Journal of Clinical Nursing, 17, 703-716.

Sharif, F., \& Masoumi, S. (2005). A qualitative study of nursing student experiences of clinical practice. BioMed Central, 4(6), 1-13.

Van Rhyn, W. J. C., \& Gontsana, M. R. (2004). Experiences by student nurses during clinical placement in psychiatric units in a hospital. Curationis, 27(4), 18-27. 